\title{
CONFIGURATION SPACES OF POINTS ON THE CIRCLE AND HYPERBOLIC DEHN FILLINGS, II
}

\author{
YASUSHI YAMASHITA, HARUKO NISHI, AND SADAYOSHI KOJIMA
}

\begin{abstract}
In our previous paper, we discussed the hyperbolization of the configuration space of $n(\geq 5)$ marked points with weights in the projective line up to projective transformations. A variation of the weights induces a deformation. It was shown that this correspondence of the set of the weights to the Teichmüller space when $n=5$ and to the Dehn filling space when $n=6$ is locally one-to-one near the equal weight. In this paper, we establish its global injectivity.
\end{abstract}

\section{INTRODUCTION}

In [7], we have shown that the configuration space of $n(\geq 5)$ marked points with weights on the real projective line up to projective transformations admits a natural hyperbolization so that the result becomes a hyperbolic cone-manifold of dimension $n-3$. In brief, we identify each component of the configuration space with the space of similarity classes of convex $n$-gons with fixed external angles in the complex plane via Schwarz-Christoffel map. Then there is a beautiful way by Thurston to hyperbolize such a space as an interior of some hyperbolic polyhedron (see [2, 6]). Each point on the boundary can be encoded by an appropriately degenerate configuration. Pasting them together along the same degenerate configurations, we obtained the resultant cone-manifold. Kapovich and Millson discussed the same hyperbolization via their duality in 5 .

The external angles can be given in fact at your choice and we regarded them as the weight. A variation of the weights induces a deformation. We restricted the set of possible external angles so that the $n$-gons are convex and can be represented as the inner polygon of the star shaped $n$-gons for any marking. More concretely, we set

$$
\Theta_{n}=\left\{\left(\theta_{1}, \ldots, \theta_{n}\right) \mid \sum_{i=1}^{n} \theta_{i}=2 \pi, \theta_{i}>0, \theta_{i}+\theta_{j}<\pi \text { for any } i \neq j\right\} .
$$

This is equivalent to say that the number of faces appeared in Thurston's polyhedralization is constant, that is $n$. Under this assumption, the topology of a deformation will be almost constant.

In [7], we discussed local behavior of the deformations appeared in our setting near the equal weight. When $n=5$, the deformations are topologically a connected sum of five copies of the real projective space, $\#^{5} \mathbf{R P}^{2}$. The assignment of the hyperbolic structure of a deformation to each weight was shown to be a local embedding at the equal weight. When $n=6$ and with the equal weight, the result of hyperbolization is a 3-dimensional hyperbolic

Date: Version 1.0 (November 26, 1998).

1991 Mathematics Subject Classification. Primary 57M50; Secondary 53C15.

Key words and phrases. hyperbolic cone-manifold, configuration space. 
manifold of finite volume with ten cusps, which we denoted by $\overline{X_{6}}$. Any deformation induced by a variation of the weights can be regarded as some Dehn filled resultant of $\overline{X_{6}}$. The assignment of the deformation to each weight was also shown to be a local embedding at the equal weight.

In this paper, we prove the global injectivity of the above assignment. Namely, we show that $\Theta_{n}$ is mapped by the above assignment injectively to the deformation space in Theorem 1 when $n=5$, and in Theorem 2 when $n=6$. The local injectivity in [7] is proven by computing the derivative of the map at the equal weight. The proof of the global injectivity we present here is based on rather geometric observation for variation of polygons developed in [6, 1], and independent of the argument in [0].

We review some of materials in [7] to set up the notations in the next section, and prove the theorems in the sections after.

\section{Preliminaries}

We here very briefly recall the hyperbolization in [7].

The configuration space of $n$ marked points in the real projective line $\mathbf{R P}^{1}$ is, by definition, the quotient of $\left(\mathbf{R P}^{1}\right)^{n}$ minus the big diagonal set by the diagonal action of the projective linear group $\operatorname{PGL}(2, \mathbf{R})$. It has $(n-1) ! / 2$ connected components, each of which is homeomorphic to a cell of dimension $n-3$. Reading off the markings of the points in counterclockwise order, each component can be labeled by a circular permutation $p=\left\langle i_{1} i_{2} \ldots i_{n}\right\rangle$ of $n$ numbers 1 to $n$ up to reversing the order.

Fix an element $\theta=\left(\theta_{1}, \ldots, \theta_{n}\right)$ of $\Theta_{n}$. Then there is a one-to-one Schwarz-Christoffel correspondence between the configuration space and the set of similarity classes $X_{n, \theta}$ of marked $n$-gons in the complex plane with external angles $\left\{\theta_{1}, \cdots, \theta_{n}\right\}$ compatible with markings (see Lemma 1 in [7]). Fix a label $p=\left\langle i_{1} i_{2} \ldots i_{n}\right\rangle$. Then the component of $X_{n, \theta}$ with label $p$ can be identified with the subset of the space of all the congruence classes of Euclidean $n$-gons with external angles $\theta_{i_{1}}, \cdots, \theta_{i_{n}}$ cyclically which consists of the ones with area 1 .

Let $x_{i}$ denote the edge of an $n$-gon starting from the vertex with angle $\theta_{i}$ in counterclockwise order, and simultaneously its length. Then, we have

$$
\sum_{j=1}^{n} x_{i_{j}} \exp \left(\sum_{k=1}^{j} \sqrt{-1} \theta_{i_{k}}\right)=0 .
$$

Let $\mathcal{E}_{p, \theta}$ be the $(n-2)$-dimensional vector space satisfying the above constrain. Then the space of congruence classes of $n$-gons is identified with the polyhedral cone $\mathcal{E}_{p, \theta} \cap \bigcap_{j=1}^{n}\left\{x_{i_{j}}>\right.$ $0\}$ in $\mathcal{E}_{p, \theta}$. The area determines a quadratic form Area of signature $(1, n-3)$ on $\mathcal{E}_{p, \theta}$ (see Lemma 2 in [7]). Thus $\mathcal{E}_{p, \theta}$ together with Area becomes a Minkowski space and Area $^{-1}(1)$ is the hyperbolic space in dimension $n-3$. Therefore the space of similarity classes of $n$-gons with fixed external angles $\theta_{i_{1}}, \cdots, \theta_{i_{n}}$ lies in the hyperbolic space bounded by the hyperbolic hyperplanes Area ${ }^{-1} \cap\left\{x_{i_{j}}=0\right\}$ for $j=1, \cdots, n$. We denote by $\Delta_{p, \theta}$ such a hyperbolic polyhedron. Then the conditions for $\theta$ as in the definition of $\Theta_{n}$ ensures us that the hyperbolic polyhedron $\Delta_{p, \theta}$ has exactly $n$ facets.

Let us denote by $\left(i_{1} i_{2}\right) i_{3} \ldots i_{n}$ or simply $\left(i_{1} i_{2}\right)$ the face of $\Delta_{p, \theta}$ represented by $\Delta_{p, \theta} \cap\left\{x_{i_{1}}=\right.$ $0\}$ since it corresponds to the degenerate configurations where the points marked by $i_{1}$ and 
$i_{2}$ collide. Similarly we use $\left(i_{1} i_{2}\right)\left(i_{3} i_{4}\right) i_{5} \ldots i_{n}$ or $\left(i_{1} i_{2} i_{3}\right) i_{4} \ldots i_{n}$, etc. to represent the codimension two faces of $\Delta_{p, \theta}$.

Now gluing $(n-1) ! / 2$ hyperbolic polyhedra $\Delta_{p, \theta}$ for all labels $p$ along the faces which represent the same degenerate configurations, we obtain $\overline{X_{n, \theta}}$ in which $X_{n, \theta}$ lies as an open dense subset.

\section{THE CASE $n=5$}

When $n=5, \Delta_{p, \theta}$ is a hyperbolic right pentagon where the edges are labeled by $\left(i_{1} i_{2}\right)$, $\left(i_{3} i_{4}\right),\left(i_{5} i_{1}\right),\left(i_{2} i_{3}\right),\left(i_{4} i_{5}\right)$ cyclically. For the equal weight $\theta_{0}=(2 \pi / 5, \cdots, 2 \pi / 5), \Delta_{p, \theta_{0}}$ is a hyperbolic regular pentagon, i.e, with all edges having equal lengths.

For any $\theta \in \Theta_{5}$, the space $\overline{X_{5, \theta}}$ is a closed hyperbolic surface homeomorphic to $\#^{5} \mathbf{R} \mathbf{P}^{2}$. The space of hyperbolic structures, which we called a Teichmüller space and denoted by $\mathcal{T}\left(\#^{5} \mathbf{R} \mathbf{P}^{2}\right)$, is parameterized by the lengths and twisting amounts for the 2 -sided ones of a maximal family of mutually disjoint nonparallel simple closed curves on $\#^{5} \mathbf{R} \mathbf{P}^{2}$. It is homeomorphic to $\mathbf{R}^{9}$. Thus we have a map $\Phi_{5}: \Theta_{5} \rightarrow \mathcal{T}\left(\#^{5} \mathbf{R} \mathbf{P}^{2}\right)$ assigning to each $\theta$ the marked hyperbolic structure of $\overline{X_{5, \theta}}$.

On the other hand, our surface $\overline{X_{5, \theta}}$ has a geometric cell decomposition into twelve hyperbolic right pentagons. The edges of the pentagons form geodesics and they are uniquely placed on $\overline{X_{5, \theta}}$ since the geodesic representative of simple closed curves within their homotopy class is unique. Hence such a cell decomposition is uniquely determined by the hyperbolic structure. In particular, the shapes of such pentagons are invariants of the hyperbolic structure on $\overline{X_{5, \theta}}$. Thus if we denote by $\mathcal{T}$ the space of all hyperbolic right pentagons, we have a map from the subset $\Phi_{5}\left(\Theta_{5}\right)$ of $\mathcal{T}\left(\#^{5} \mathbf{R} \mathbf{P}^{2}\right)$ to the direct product of twelve $\mathcal{T}$ 's by listing hyperbolic structures of $\Delta_{p, \theta}$ 's. Here is a part of our surface (Fig. 1).

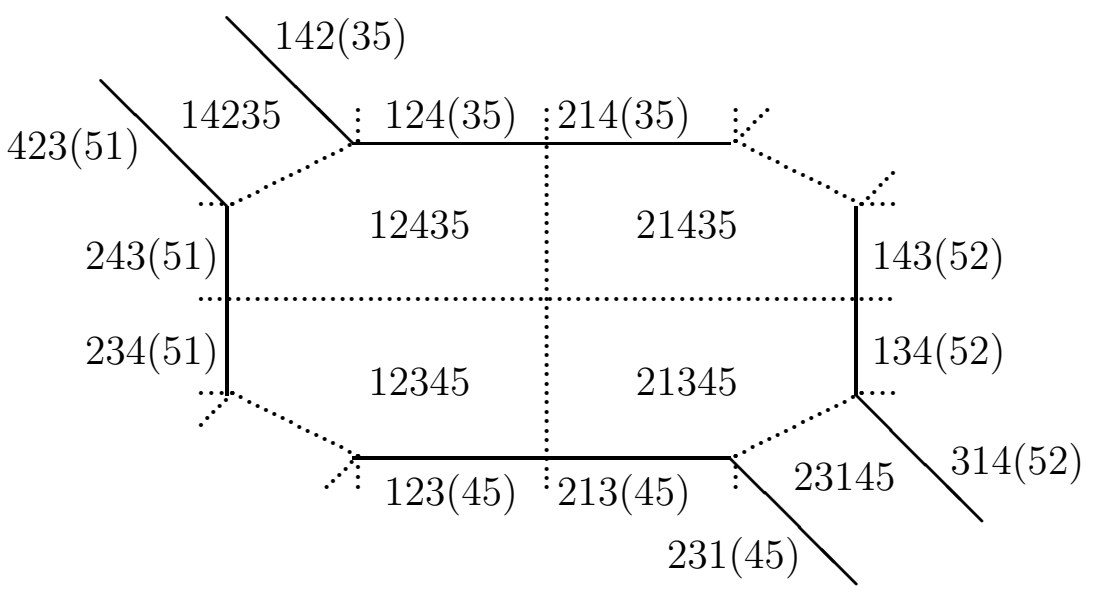

FiguRE 1.

A hyperbolic right pentagon is determined by lengths of two adjacent sides. The parameterization of the space $\mathcal{T}$ can be given as follows. Put a hyperbolic right pentagon in the projective model on the unit disk so that it lies in the first quadrant and the preferred two adjacent sides are on the axes. Let $P$ and $Q$ be the Euclidean lengths of the two sides on 
the axes. Then these are subject to the relation $P^{2}+Q^{2}>1$. Conversely if we are given such $P<1$ and $Q<1$, we can construct a unique hyperbolic pentagon. Thus $\mathcal{T}$ can be identified with the set

$$
\left\{(P, Q) \mid 0<P, Q<1, P^{2}+Q^{2}>1\right\} .
$$

We now review from [6] how the lengths $P$ and $Q$ for the hyperbolic right pentagon $\Delta_{p, \theta}$ can be computed in a geometric way where $p=\left\langle i_{1} i_{2} i_{3} i_{4} i_{5}\right\rangle$. First recall that the space $\mathcal{E}_{p, \theta}$ of congruence classes of Euclidean (extended) pentagons with external angles $\theta_{i_{1}}, \ldots, \theta_{i_{5}}$ cyclically for $\theta=\left(\theta_{1}, \ldots, \theta_{5}\right)$ is a 3 -dimensional Lorentz space. Its coordinate $(x, u, v)$ can be given by setting

$$
x=\sqrt{\operatorname{Area} T_{0}}, \quad u=\sqrt{\operatorname{Area} T_{1}}, \quad v=\sqrt{\operatorname{Area} T_{2}},
$$

where $T_{0}$ is a triangle obtained by completing the pentagon $H$ by extending the edges $x_{i_{2}}$, $x_{i_{4}}$ and $x_{i_{5}}$, and $T_{1}, T_{2}$ the yielding triangles adjacent to the edge $x_{i_{1}}, x_{i_{3}}$ respectively (see Fig. 2).

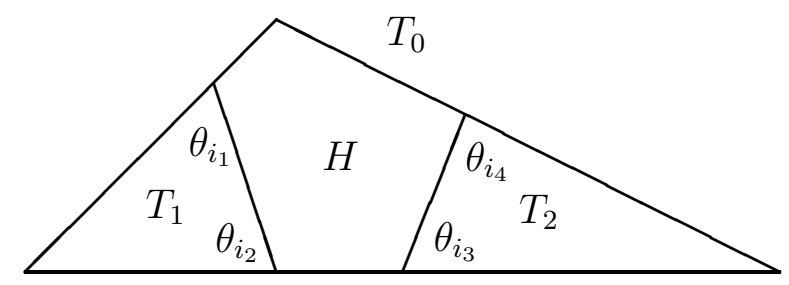

FIGURE 2.

Then $\Delta_{p, \theta}$ is a hyperbolic right pentagon lying in the hyperbola $\operatorname{Area}^{-1}(1)$ in $\mathcal{E}_{p, \theta}$. Projecting it along the rays towards the origin into the unit disk of the plane $\{x=1\}$, we represent it in the projective model with $(u, v)$-coordinate. Then we see that $\Delta_{p, \theta}$ lies in the first quadrant of the $u v$-plane with the edge $\left(i_{1} i_{2}\right) i_{3} i_{4} i_{5}$ lying on the $u$-axis and $i_{1} i_{2}\left(i_{3} i_{4}\right) i_{5}$ on the $v$-axis. Let $(P, 0)$ be the Euclidean coordinates of the intersection of the adjacent edges $\left(i_{1} i_{2}\right) i_{3} i_{4} i_{5}$ and $i_{1} i_{2} i_{3}\left(i_{4} i_{5}\right)$, and $(0, Q)$ the intersection of adjacent edges $i_{1} i_{2}\left(i_{3} i_{4}\right) i_{5}$ and $\left(i_{5} i_{1}\right) i_{2} i_{3} i_{4}$. To compute $P$, consider the degenerate pentagon $H_{P}$ with $x_{i_{1}}=x_{i_{4}}=0$ in $T_{0}$ so that $T_{0}$ consists of $H_{P}$ and $T_{2}^{e}$, and for $Q$ consider the degenerate pentagon $H_{Q}$ with degenerate sides $x_{i_{5}}=x_{i_{3}}=0$ in $T_{0}$ so that $T_{0}$ consists of $H_{Q}$ and $T_{1}^{e}$ (see Fig. 3). Then by the definition of the coordinates of $\mathcal{E}_{p, \theta}$ and recalling that we are in the projective
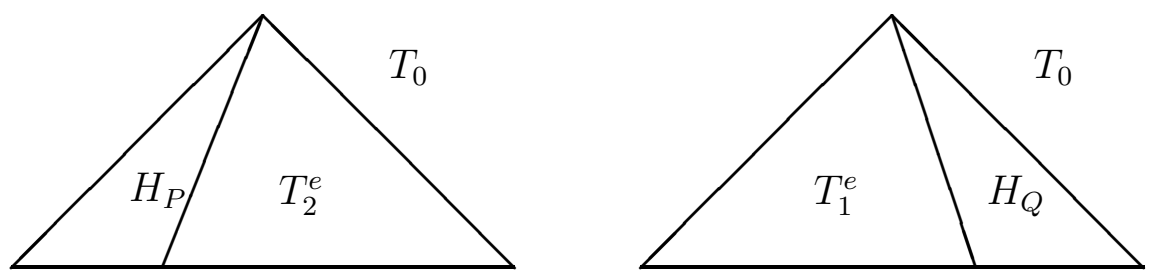

FiguRE 3. 
model in the plane $x=1$, we obtain

and

$$
P^{2}=\frac{\text { Area } T_{2}^{e}}{\text { Area } T_{0}}=\frac{\text { base width of } T_{2}^{e}}{\text { base width of } T_{0}}
$$

$$
Q^{2}=\frac{\text { Area } T_{1}^{e}}{\text { Area } T_{0}}=\frac{\text { base width of } T_{1}^{e}}{\text { base width of } T_{0}}
$$

Therefore by normalizing $T_{0}$ so that its base width has length 1 , the quantities $P^{2}$ and $Q^{2}$ are depicted just on the base edge of $T_{0}$ as in Fig. 4 .

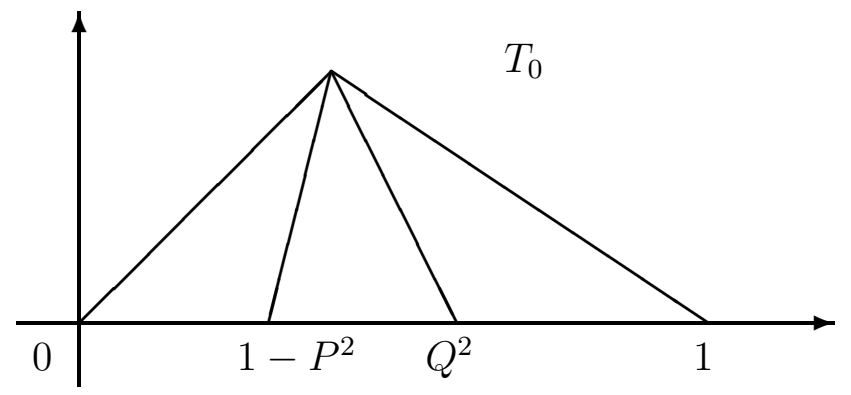

FigURE 4.

Now let $\Psi_{p}: \Theta_{5} \rightarrow \mathcal{T}$ be the map sending $\theta \in \Theta_{5}$ to the hyperbolic right pentagon $\Delta_{p, \theta}$. Then the following is shown in [6].

Lemma 1. The inverse image of a point in $\mathcal{T}$ by $\Psi_{p}$ is in one-to-one correspondence with the upper halfplane $\mathbf{H}^{2}$.

We review here the proof in [6] for reader's convenience.

Proof. Suppose $(P, Q) \in \mathcal{T}$. For a point $w$ in $\mathbf{H}^{2}$, plot four points $0<1-P^{2}<Q^{2}<1$ on the real axis of $\mathbf{C}$ and join these points with $w$ (Fig. 51). We denote these four edges by $e_{0}, e_{P}, e_{Q}, e_{1}$ as in the figure. Slide $e_{P}$ and $e_{Q}$ to the points 1 and 0 respectively so that the lines are parallel to the originals and do not intersect with each other. Then these new two edges together with $e_{0}, e_{1}$ and the real-axis form a pentagon with its external angles $\left(\theta_{i_{1}}, \ldots, \theta_{i_{5}}\right)$ as in Fig. 5 . Then by the argument above, we see that $\theta=\left(\theta_{1}, \ldots, \theta_{5}\right)$ satisfies $\Psi_{p}(\theta)=(P, Q)$. Since the choice of $w$ is arbitrary in $\mathbf{H}^{2}$, by checking that the different $w$ gives different $\theta$ we get the conclusion.

Now consider the product map $\Psi_{\langle 12345\rangle} \times \Psi_{\langle 21435\rangle}: \Theta_{5} \rightarrow \mathcal{T} \times \mathcal{T}$ which sends $\theta$ to $\left(\Delta_{\langle 12345\rangle, \theta}, \Delta_{\langle 21435\rangle, \theta}\right)$. Then we claim that

Lemma 2. The map $\Psi_{\langle 12345\rangle} \times \Psi_{\langle 21435\rangle}$ is injective.

Proof. Let $\left(\left(P_{1}, Q_{1}\right),\left(P_{2}, Q_{2}\right)\right)$ be any element of the image of $\Psi_{\langle 12345\rangle} \times \Psi_{\langle 21435\rangle}$, that is, $P_{1}, Q_{1}, P_{2}, Q_{2}$ are the lengths of the sides (12)345, 12(34)5, (21)435, 21(43)5, respectively. Suppose that $\theta=\left(\theta_{1}, \ldots, \theta_{5}\right)$ be any element in the inverse image of $\left(\left(P_{1}, Q_{1}\right),\left(P_{2}, Q_{2}\right)\right)$ by $\Psi_{\langle 12345\rangle} \times \Psi_{\langle 21435\rangle}$. Then $\theta$ lies in the intersection $\Psi_{\langle 12345\rangle}^{-1}\left(\left(P_{1}, Q_{1}\right)\right) \cap \Psi_{\langle 21435\rangle}^{-1}\left(\left(P_{2}, Q_{2}\right)\right)$. Let $w_{1}$ be the point in $\mathbf{H}^{2} \cong \Psi_{\langle 12345\rangle}^{-1}\left(\left(P_{1}, Q_{1}\right)\right)$ corresponding to $\theta$ under the identification in 


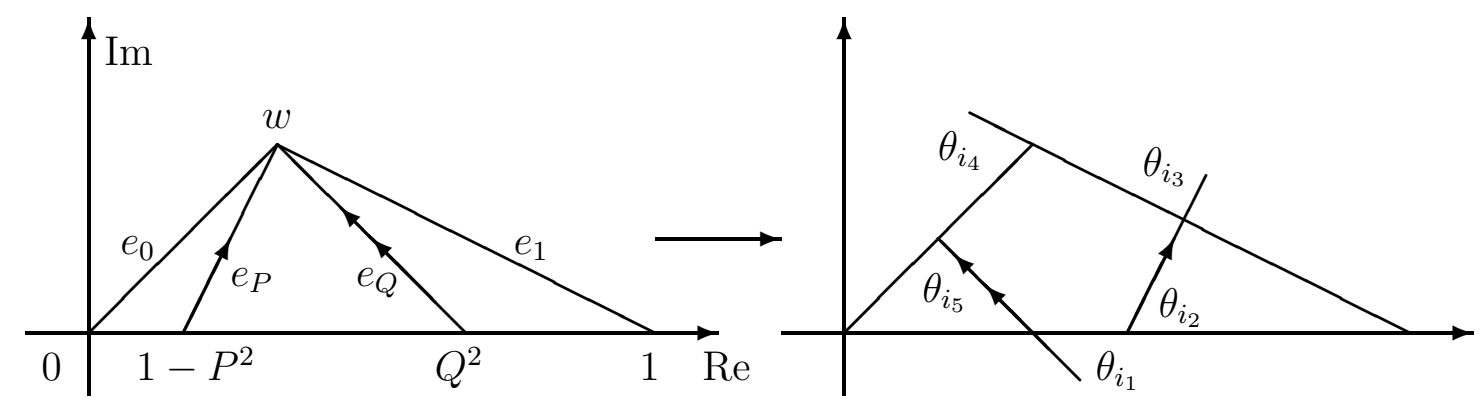

FiguRe 5. Map from $w$ to $\theta$

Lemma 1, and similarly $w_{2}$ the point in $\mathbf{H}^{2} \cong \Psi_{\langle 21435\rangle}^{-1}\left(\left(P_{2}, Q_{2}\right)\right)$ corresponding to $\theta$. These can be obtained by the inverse procedure of Fig. 5. Then we see that the triangles $\triangle w_{1} 01$ and $\triangle w_{2} 01$ are congruent since both have the external angles $\theta_{5}, \theta_{1}+\theta_{2}, \theta_{3}+\theta_{4}$. Thus $w_{1}$ and $w_{2}$ must be identical in the upper half plane $\mathbf{H}^{2}$. Let $w=w_{1}=w_{2}$.

The angle at $P_{1}^{2}$ in the triangle $\triangle w 0 P_{1}^{2}$ is $\theta_{2}$, and the angle at $P_{2}^{2}$ in the triangle $\triangle w 0 P_{2}^{2}$ is $\theta_{1}$, so $\triangle w 0 P_{1}^{2}$ and $\triangle P_{2}^{2} 0 w$ are similar. (See Fig. 6). Then we have

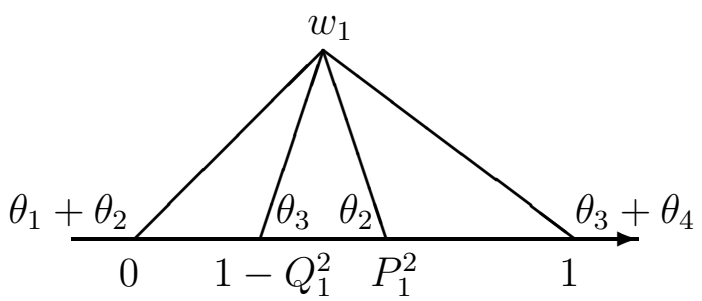

(a)

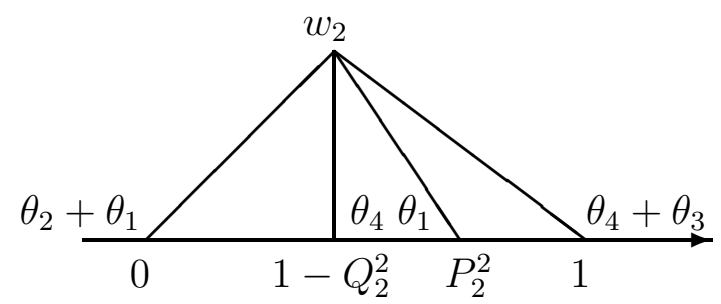

(b)

FiguRE 6.

$$
|w|: P_{1}^{2}=P_{2}^{2}:|w|
$$

Therefore

$$
|w|^{2}=\left(P_{1} P_{2}\right)^{2} .
$$

This implies that $w$ must lie on the circle with center 0 and radius $P_{1} P_{2}$. In the same way, since the triangles $\triangle w_{1}\left(1-Q_{1}^{2}\right) 1$ and $\triangle\left(1-Q_{2}^{2}\right) 1 w_{2}$ are similar, we see that $w$ must lie on the circle with center 1 and radius $Q_{1} Q_{2}$. Since the above two circles intersect at one point on the upper halfplane, the point $w$, therefore $\theta$, is uniquely determined.

By this lemma, we see that the composition assigning $\theta \mapsto \overline{X_{5, \theta}} \mapsto\left(\Delta_{\langle 12345\rangle, \theta}, \Delta_{\langle 21435\rangle, \theta}\right)$ is injective, and hence we have proven the following.

Theorem 1. The map $\Phi_{5}$ from $\Theta_{5}$ to $\mathcal{T}\left(\#^{5} \mathbf{R} \mathbf{P}^{2}\right)$ defined by the assignment to $\theta$ the hyperbolic structure of $\overline{X_{5, \theta}}$ is injective. 


\section{THE CASE $n=6$}

When $n=6, \Delta_{p, \theta}$ is a hyperbolic hexahedron bounded by six faces $\left(i_{1} i_{2}\right),\left(i_{2} i_{3}\right) \ldots,\left(i_{6} i_{1}\right)$ with having two vertices $\left(i_{1} i_{2}\right)\left(i_{3} i_{4}\right)\left(i_{5} i_{6}\right)$ and $\left(i_{2} i_{3}\right)\left(i_{4} i_{5}\right)\left(i_{6} i_{1}\right)$ at finite distance where three faces $\left(i_{k} i_{k+1}\right)$ are coming and meeting orthogonally each other for $k=1,3,5$ and $k=2,4,6$ respectively. Moreover, the faces $\left(i_{k} i_{k+1}\right)$ and $\left(i_{k+3} i_{k+4}\right)$ always intersect orthogonally for $k=1,2,3$. For the equal weight $\theta_{0}=(2 \pi / 6, \cdots, 2 \pi / 6)$, the dihedral angle between the faces $\left(i_{k} i_{k+1}\right)$ and $\left(i_{k+1} i_{k+2}\right)$ is zero for $k=1, \ldots, 6$ so that $\Delta_{p, \theta_{0}}$ has three ideal vertices. For other weights $\theta \neq \theta_{0}$, the faces $\left(i_{k} i_{k+1}\right)$ and $\left(i_{k+1} i_{k+2}\right)$ intersect if and only if $\theta_{i_{k}}+\theta_{i_{k+1}}+\theta_{i_{k+2}}<\pi$. Note that in such a case, $\left(i_{k+3} i_{k+4}\right)$ and $\left(i_{k+4} i_{k+5}\right)$ don't intersect since $\theta_{i_{k+3}}+\theta_{i_{k+4}}+\theta_{i_{k+5}}>\pi$.

The hyperbolized configuration space of the equal weight in this case is a complete hyperbolic 3-manifold $\overline{X_{6}}$ of finite volume with ten cusps. A perturbation gives rise to not a deformation in the usual sense, but a resultant of hyperbolic Dehn filling. The space of hyperbolic Dehn fillings of a complete hyperbolic 3-manifold can be locally identified with the space of representations of a fundamental group up to conjugacy. It has a structure of a smooth algebraic variety of complex dimension $=$ the number of cusps, ([3], [9], [8], [4]). Hence in our case, the space of Dehn fillings $\mathcal{H}\left(\overline{X_{6}}\right)$ is locally biholomorphic to $\mathbf{C}^{10}$.

Here is a part of our 3 -manifold $\overline{X_{6}}$ in the projective model (Fig. [). The figure illustrates

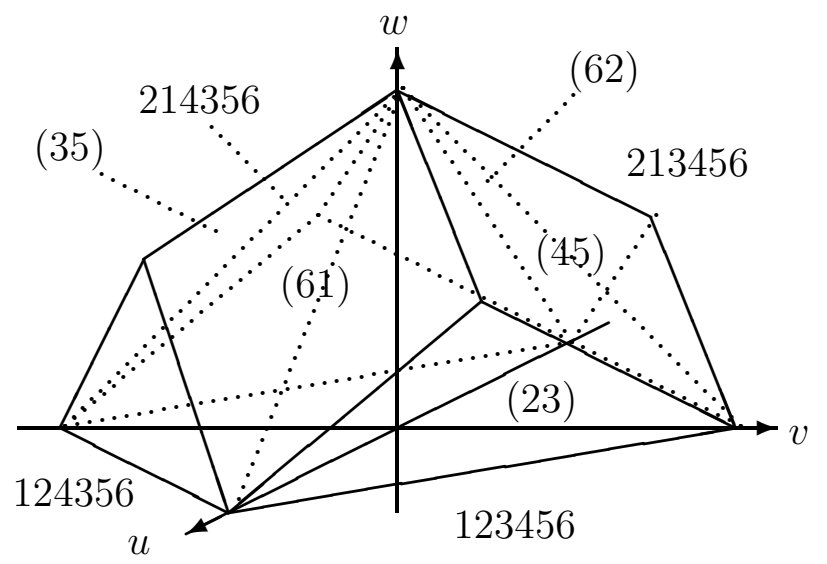

Figure 7.

the union of four hexahedra in the case of the equal weight. The vertices on the axes are located on the ideal boundary, but other four vertices are at finite distance. Six planes $u=0, v=0, w=0, u+v=1, v+w=1$ and $w+u=1$ correspond to the collision of (12), (34), (56), (23), (45) and (61) respectively. The origin corresponds to (12)(34)(56).

If we perturb the weight to $\theta$ so that $\theta_{i_{k}}+\theta_{i_{k+1}}+\theta_{i_{k+2}}<\pi$, then three vertices $i_{k}, i_{k+1}$, $i_{k+2}$ of the hexagon can collide, and $\left(i_{k} i_{k+1}\right)$ and $\left(i_{k+1} i_{k+2}\right)$ intersects to yield a new edge. The dihedral angles about the old edges are $\pi / 2([\overline{7})$ so that the bounding planes form geodesic surfaces. But the dihedral angle at the new edge depends on the weight $\theta$ and it creates a cone-type singularity in $\overline{X_{6, \theta}}$ so that $\overline{X_{6, \theta}}$ becomes a hyperbolic cone-manifold. Notice that the complement of the cone-singularity is homeomorphic to $\overline{X_{6}}$ but endowed with an incomplete hyperbolic structure. Then $\overline{X_{6, \theta}}$ can be regarded as the resultant of 
the hyperbolic Dehn filling of the complete hyperbolic 3-manifold $\overline{X_{6}}$, which lies in $\mathcal{H}\left(\overline{X_{6}}\right)$ (for more detail, see [7]). Thus we have a map $\Phi_{6}: \Theta_{6} \rightarrow \mathcal{H}\left(\overline{X_{6}}\right)$ assigning to each $\theta$ the hyperbolic cone-manifold $\overline{X_{6, \theta}}$.

There are 15 geodesic surfaces in $\overline{X_{6, \theta}}$ represented by a pair of markings $(i j)$ where $i \neq j \in\{1,2, \ldots, 6\}$. They are uniquely placed on $\overline{X_{6, \theta}}$ since the geodesic representative of a surface within their proper homotopy class is unique if any. Hence a geometric cell decomposition by such surfaces, which consists of 60 hexahedra, is uniquely determined and the shapes of these hexahedra $\Delta_{p, \theta}$ are invariants of the hyperbolic (cone) structure on $\overline{X_{6, \theta}}$. Let $\mathcal{H}$ be the space of all hexahedra which have the property as $\Delta_{p, \theta}$ described in the first paragraph of this section. Then we have a map from the subset $\Phi_{6}\left(\Theta_{6}\right)$ of $\mathcal{H}$ to the direct product of sixty $\mathcal{H}$ 's by listing hyperbolic structures of $\Delta_{p, \theta}$ 's.

The parameterization of the space $\mathcal{H}$ can be given as follows. First let us make the definition of $\mathcal{H}$ precise. We use the notations of the faces or vertices of the elements in $\mathcal{H}$ as $\Delta_{p, \theta}$ where $p=\langle 123456\rangle$. Then $\mathcal{H}$ is the set of all hyperbolic hexahedra having the following properties; (i) it is bounded by six faces $(12),(23) \ldots,(61)$, (ii) the three faces $(k k+1)$ meet orthogonally each other for $k=1,3,5$ and $k=2,4,6$ to make vertices $(12)(34)(56)$ and $(23)(45)(61)$ at finite distance, respectively, (iii) the faces $(k k+1)$ and $(k+3 k+4)$ always meet orthogonally for $k=1,2,3$.

We present a hyperbolic hexahedron in $\mathcal{H}$ in the projective model located in the unit ball in the Euclidean 3-space with uvw-coordinates so that the vertex $(12)(34)(56)$ is at the origin, the faces (12), (34), (56) are lying on the $v w, w u, u v$-planes, respectively. Since the other three faces (45), (61) and (23) are orthogonal in a hyperbolic sense to the faces (12), (34) and (56) respectively, they are orthogonal also in an Euclidean sense in this model there. We denote the hyperplane on which the face $(i j)$ lies by the same symbol $(i j)$, for simplicity.

Let $(P, 0,0)$ be the Euclidean coordinates of the intersection of the plane (61) with $u$ axis, $(0, Q, 0)$ the intersection of $(23)$ with $v$-axis, $(0,0, R)$ the intersection of (45) with $w$-axis, respectively. Then depending on the signs of $P-1, Q-1, R-1$, there are several combinatorial types of hexahedra in $\mathcal{H}$ (see Fig. 8.) To describe each type, it is enough to look at the cases $(a) P<1, Q<1, R<1$ and $(b) P>1, Q<1, R<1$. Let $p=\operatorname{mim}\{P, 1 / P\}, q=\operatorname{mim}\{Q, 1 / Q\}, r=\operatorname{mim}\{R, 1 / R\}$.

Case $(a) . P<1, Q<1, R<1$;

In this case, since the face (61) intersects with (56), the face (23) with (12), the face (45) with (34) within the unit ball in the $u v w$-space respectively, all the faces of $\Delta_{p, \theta}$ are quadrilaterals with three right angles. (See Fig. $8(a)$.) Then $p=P, q=Q, r=R$, and the points $(p, 0,0),(0, q, 0),(0,0, r)$ are the vertices of the hexahedron.

Case $(b) . P>1, Q<1, R<1$;

In this case, the plane (61) does not intersect with (56) inside the unit ball, while the faces (23) and (34) intersect. (See Fig. $8(b)$ ). Then the face (34) is a hyperbolic right pentagon, whose side on the $u$-axis has the Euclidean length $p=1 / P$. This is because when restricted to the hyperbolic 2-plane $\{v=0\}$, the point $(P, 0,0)$ is the intersection of the two lines extending the edges $(61) \cap(34)$ and $(34) \cap(56)$ respectively, both of which are orthogonal to the edge $(23) \cap(34)$. In other words, the point $(P, 0,0)$ is the dual of the line containing the edge $(23) \cap(34)$ (see Fig 9). The shape of the faces (23) is also a hyperbolic pentagon, 

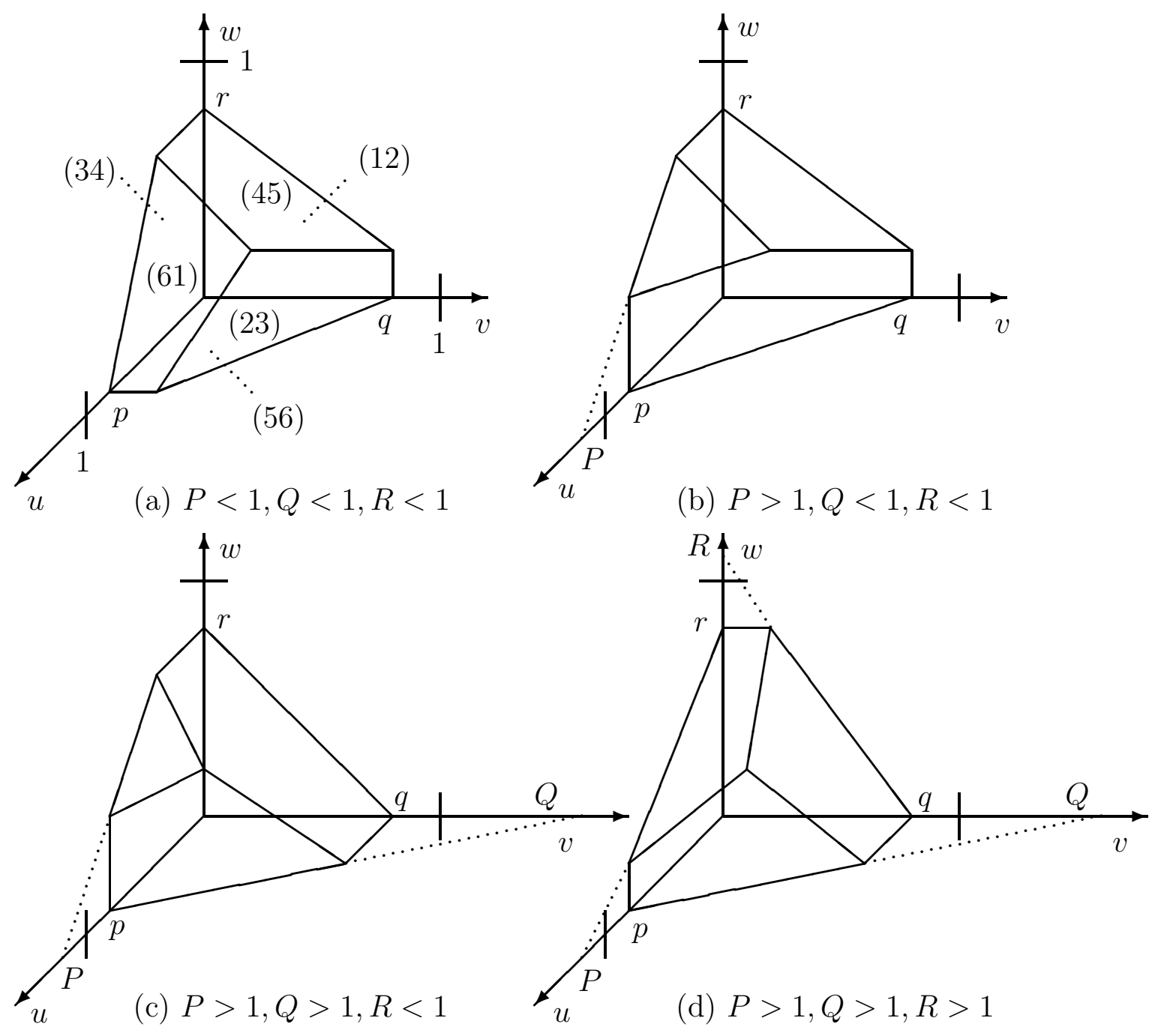

FiguRE 8.

the faces (12) and (45) are hyperbolic quadrilaterals with three right angles, and the faces (61) and (56) are hyperbolic right triangles.

Note that when $P=1, Q<1, R<1$, the hexahedra has an ideal vertex at $(1,0,0)$. This means that the faces (61) and (56) do not meet, but they are tangent at $(1,0,0)$, and also (23) and (34) are tangent at $(1,0,0)$. So the face (61) and the face (56) are hyperbolic right triangles with one ideal vertex, the face (23) and the face (34) quadrilaterals with one ideal vertex and three right angles. The faces (12) and (45) are as in the case $(a)$ or $(b)$.

Now conversely when three numbers $P, Q, R>0$ are given, by taking the inverse procedure above, we can construct a unique hyperbolic hexahedron in $\mathcal{H}$. That is, its combinatorial type is determined by the signs of $P-1, Q-1, R-1$, and the lengths of the edges on the $u, v, w$-axes are $p, q, r$, respectively. Since each face is either a hyperbolic right pentagon, a quadrilateral with three right angles (possibly with one ideal vertices), 


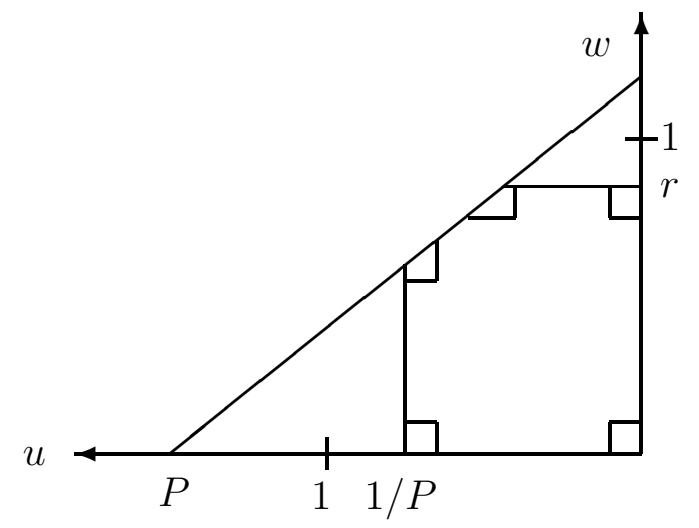

FIGURE 9.

or a hyperbolic right triangle (possibly with ideal vertices), all the other lengths of the hexahedron are uniquely determined and the vertex where (61), (23) and (45) meet is at finite distance. Therefore $\mathcal{H}$ can be identified with the set

$$
\{P, Q, R \mid P, Q, R>0\} .
$$

Following [1], we shall show a geometric way of computing the parameters $P, Q, R$ for the hyperbolic hexahedron $\Delta_{p, \theta}$ as in the case $n=5$.

Recall that for $p=\left\langle i_{1} i_{2} i_{3} i_{4} i_{5} i_{6}\right\rangle$ the space $\mathcal{E}_{p, \theta}$ of hexagons with external angles $\theta_{i_{1}}, \ldots$, $\theta_{i_{6}}$ cyclically is a Lorentz space with coordinates $(x, u, v, w)$ given by

$$
x=\sqrt{\operatorname{Area} T_{0}}, \quad u=\sqrt{\operatorname{Area} T_{1}}, \quad v=\sqrt{\operatorname{Area} T_{2}}, \quad w=\sqrt{\operatorname{Area} T_{3}},
$$

where $T_{0}$ is a triangle obtained by completing a convex hexagon by extending the edges $x_{i_{2}}$, $x_{i_{4}}, x_{i_{6}}$, and $T_{1}, T_{2}, T_{3}$ are yielding triangles adjacent to the edges $x_{i_{1}}, x_{i_{3}}, x_{i_{5}}$, respectively as in Fig. 10. Then $\Delta_{p, \theta}$ is a hyperbolic hexahedron in a hyperbola $\operatorname{Area}^{-1}(1)$ in $\mathcal{E}_{p, \theta}$.

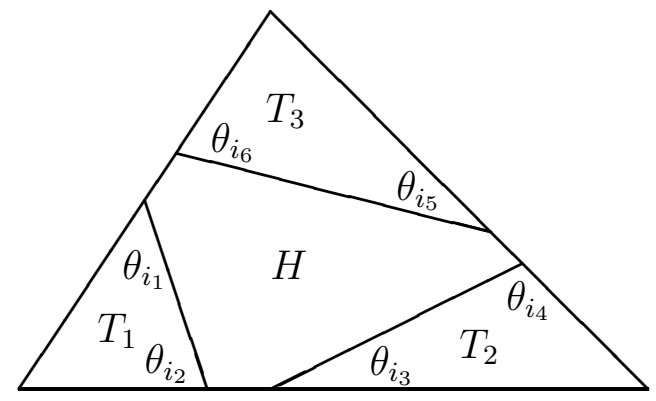

FiguRE 10 .

By projecting it along the rays towards the origin into the unit ball in $\{x=1\}$, we get $\Delta_{p, \theta}$ in the projective model with $(u, v, w)$-coordinate. Then $\Delta_{p, \theta}$ lies in the first orthant $\{u, v, w \mid u \geq 0, v \geq 0, w \geq 0\}$ with the vertex $\left(i_{1} i_{2}\right)\left(i_{3} i_{4}\right)\left(i_{5} i_{6}\right)$ at the origin and the faces of $\Delta_{p, \theta}$ labeled by $\left(i_{1} i_{2}\right),\left(i_{3} i_{4}\right)$ and $\left(i_{5} i_{6}\right)$ lying in the planes $\{u=0\},\{v=0\}$ and $\{w=0\}$, respectively. 
The parameters $P, Q, R$ for the hexahedron $\Delta_{p, \theta} \in \mathcal{H}$ can be computed as follows. Since $(P, 0,0)$ is the intersection of the plane $\left(i_{6} i_{1}\right)$ with the $u$-axis, $(0, Q, 0)$ the intersection of the plane $\left(i_{2} i_{3}\right)$ with $v$-axis, and $(0,0, R)$ the intersection of the plane $\left(i_{4} i_{5}\right)$ with $w$-axis, the points $(P, 0,0),(0, Q, 0)$ and $(0,0, R)$ correspond to the degenerate hexagons with the sides $x_{i_{3}}=x_{i_{5}}=x_{i_{6}}=0, x_{i_{1}}=x_{i_{2}}=x_{i_{5}}=0$ and $x_{i_{1}}=x_{i_{3}}=x_{i_{4}}=0$ respectively.

Let $a, b, c$ be the vertices of $T_{0}$ at which the external angles are $\theta_{i_{1}}+\theta_{i_{2}}, \theta_{i_{3}}+\theta_{i_{4}}, \theta_{i_{5}}+\theta_{i_{6}}$, respectively, and $c^{\prime}, b^{\prime}, a^{\prime}$ the points on the (possibly extended) edges $a b, b c, c a$ such that the segments $c c^{\prime}, a a^{\prime}, b b^{\prime}$ are parallel to the edges $x_{i_{1}}, x_{i_{3}}, x_{i_{5}}$ respectively (see Fig.11). Then by the similar procedure of presenting such degenerate hexagons in the triangle $T_{0}$

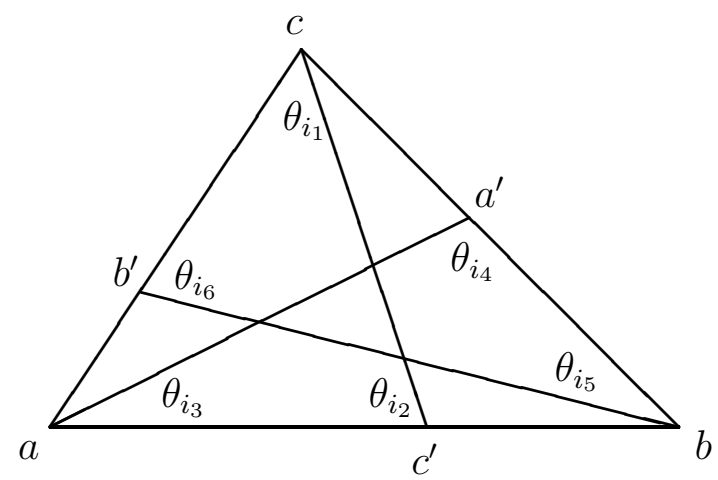

FiguRE 11.

as in the case $n=5$, we obtain

$$
\begin{aligned}
& P^{2}=\frac{\text { Area } \triangle a c^{\prime} c}{\text { Area } T_{0}}=\frac{\text { length of } a c^{\prime}}{\text { length of } a b}, \\
& Q^{2}=\frac{\text { Area } \triangle b a^{\prime} a}{\text { Area } T_{0}}=\frac{\text { length of } b a^{\prime}}{\text { length of } b c}, \\
& R^{2}=\frac{\text { Area } \triangle c b^{\prime} b}{\text { Area } T_{0}}=\frac{\text { length of } c b^{\prime}}{\text { length of } c a} .
\end{aligned}
$$

Thus we can compute $P, Q, R$ by just looking at the Fig.11. We note that the signs of $P-1, Q-1, R-1$ are the same as those of $\theta_{i_{5}}+\theta_{i_{6}}+\theta_{i_{1}}-\pi, \theta_{i_{1}}+\theta_{i_{2}}+\theta_{i_{3}}-\pi$, $\theta_{i_{3}}+\theta_{i_{4}}+\theta_{i_{5}}-\pi$ respectively (see Fig.12).

Now let $\Psi_{p}: \Theta_{6} \rightarrow \mathcal{H}$ be the map sending $\theta \in \Theta_{6}$ to the hyperbolic hexahedron $\Delta_{p, \theta} \in \mathcal{H}$. Then, as in the case $n=5$, the following result is proven in [1], where we shall give a proof for reader's convenience.

Lemma 3. For a label $p=\left\langle i_{1} i_{2} \ldots i_{6}\right\rangle$, the inverse image of a point in $\mathcal{H}$ by $\Psi_{p}$ is in one-to-one correspondence with the upper half plane $\mathbf{H}^{2}$.

Proof. Let $w$ be in $\mathbf{H}^{2}$. Together with the point 0 and 1 , it forms a triangle which we denote by $T$. We add three edges in $T$. Let $X$ be the point $P^{2}$ in the complex plane and add new edge $w X$. Likewise, let $Y$ be $1+(w-1) Q^{2}$ and join 0 and $Y$. And last, let $Z$ be $w\left(1-R^{2}\right)$ and join 1 and $Z$. By sliding the newly added edges like in the case $n=5$, we get 


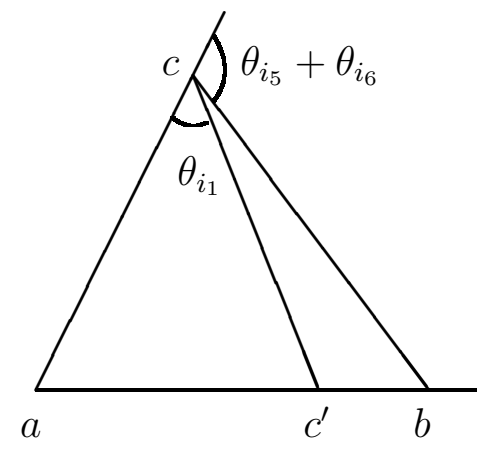

(a) $P<1$

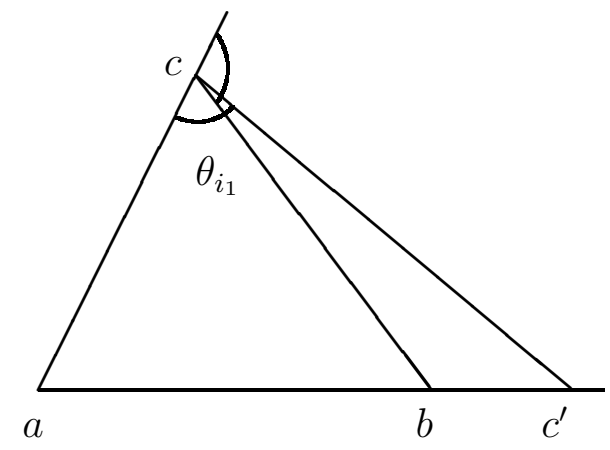

(b) $P>1$

FIGURE 12.

six angles $\theta_{i_{1}}, \ldots, \theta_{i_{6}}$. For the edge with endpoints $X$, the direction to slide is toward 0 . For $Y$ and $Z$, it is 1 and $w$ respectively (see Fig. 13). Then by the argument above, we see

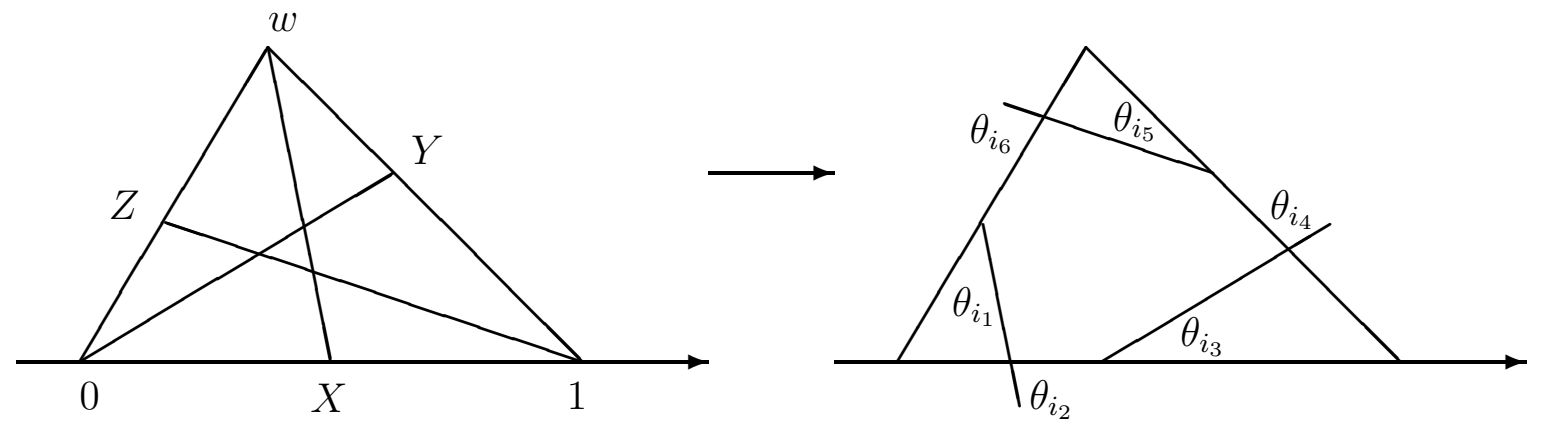

Figure 13. Map from $w$ to $\theta$

that $\Psi_{p}\left(\left(\theta_{1}, \ldots, \theta_{6}\right)\right)=(P, Q, R)$. Since $w$ is arbitrary in $\mathbf{H}^{2}$, we get the conclusion.

Consider now the map $\Psi_{\langle 123456\rangle} \times \Psi_{\langle 214356\rangle}: \Theta_{6} \rightarrow \mathcal{H} \times \mathcal{H}$ which sends $\theta$ to $\left(\Delta_{\langle 123456\rangle, \theta}\right.$, $\left.\Delta_{\langle 214356\rangle, \theta}\right)$. Then these two hexahedra can be seen in Fig. 7 stand on the first and third quadrants of the $u v$-plane. Notice that they have the common edge (12)(34)56 along the $w$-axis. Then we claim the following.

Lemma 4. The map $\Psi_{\langle 123456\rangle} \times \Psi_{\langle 214356\rangle}$ is injective.

Proof. Let $\left(\left(P_{1}, Q_{1}, R_{1}\right),\left(P_{2}, Q_{2}, R_{2}\right)\right)$ be an element in the image of $\Psi_{\langle 123456\rangle} \times \Psi_{\langle 214356\rangle}$ and $\theta=\left(\theta_{1}, \ldots, \theta_{6}\right)$ any element in the preimage of $\left(\left(P_{1}, Q_{1}, R_{1}\right),\left(P_{2}, Q_{2}, R_{2}\right)\right)$. Set $w_{1}$ and $w_{2}$ the points in $\xi_{\langle 123456\rangle}^{-1}\left(\left(P_{1}, Q_{1}, R_{1}\right)\right)$ and $\xi_{\langle 214356\rangle}^{-1}\left(\left(P_{2}, Q_{2}, R_{2}\right)\right)$ corresponding to $\theta$ under the identification in Lemma 3 respectively. Again, we see that the triangles $\triangle w_{1} 01$ and $\triangle w_{2} 01$ are congruent so that $w_{1}$ and $w_{2}$ are identical which we denote by $w$.

Let $X_{i}=P_{i}^{2}, Y_{i}=1+(w-1) Q_{i}^{2} \in \mathbf{H}^{2}$ for $i=1,2$. Then the triangles $\triangle 0 X_{1} w$ and $\triangle 0 w X_{2}$ are similar since both have the external angles $\theta_{i_{1}}+\theta_{i_{2}}, \pi-\theta_{i_{2}}, \pi-\theta_{i_{1}}$. Similarly, the triangles $\triangle 10 Y_{1}$ and $\triangle 1 Y_{2} 0$ are similar since both of which have the external angles $\theta_{i_{3}}+\theta_{i_{4}}, \pi-\theta_{i_{3}}, \pi-\theta_{i_{4}}$. (see Fig. 14). Thus we have 


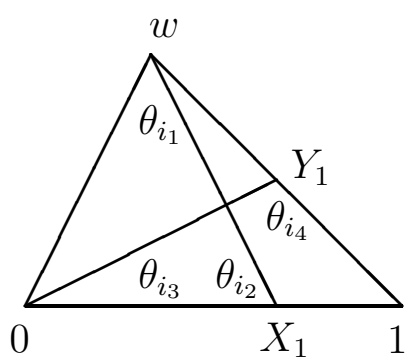

(a)

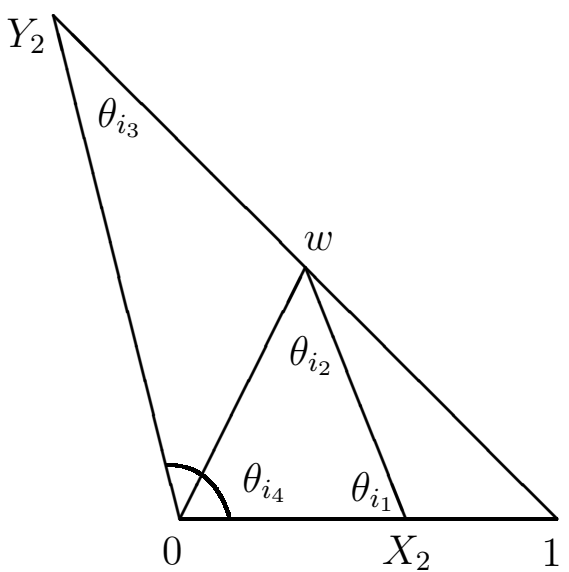

(b)

FiguRE 14.

$$
P_{1}^{2}:|w|=|w|: P_{2}^{2}
$$

and

$$
1:|w-1| Q_{1}^{2}=|w-1| Q_{2}^{2}: 1
$$

Therefore $w$ is uniquely determined as the point on the upper half plane where the two circles $|z|^{2}=\left(P_{1} P_{2}\right)^{2}$ and $|z-1|^{2}=1 /\left(Q_{1} Q_{2}\right)^{2}$ meet. And so we conclude that $\theta$ is unique.

By the above lemma, we have proven the following theorem.

Theorem 2. The map $\Phi_{6}$ from $\Theta_{6}$ to the space of Dehn fillings $\mathcal{H}\left(\overline{X_{6}}\right)$ defined by assigning to $\theta$ the hyperbolic cone manifold $\overline{X_{6, \theta}}$ is injective.

\section{REFERENCES}

[1] K. Ahara and K. Yamada, Shapes of Hexagrams, preprint, Meiji University, 1997.

[2] C. Bavard and E. Ghys, Polygones du plan et polyedres hyperboliques, Geom. Dedicate 43 (1992), 207-224.

[3] M. Culler and P. Shalen, Varieties of group representations and splittings of 3-manifolds, Ann. of Math., 117 (1983), 109-146.

[4] C. Hodgson and S. Kerckhoff, Rigidity of hyperbolic cone-manifolds and hyperbolic Dehn surgery, J. Differential Geom., 48 (1998), 1-59.

[5] M. Kapovich and J. Millson, On the moduli space of polygons in the Euclidean plane, J. Differential Geom., 42 (1995), 133-164.

[6] S. Kojima and Y. Yamashita, Shapes of stars, Proc. Amer. Math. Soc., 117 (1993), 845-851.

[7] S. Kojima, H. Nishi and Y. Yamashita, Configuration spaces of points on the circle and hyperbolic Dehn fillings, to appear in Topology.

[8] W. Neumann and D. Zagier, Volumes of hyperbolic 3-manifolds, Topology, 24 (1985), 307-332.

[9] W. Thurston, Geometry and Topology of 3-manifolds, Lecture Notes, Princeton Univ., 1977/78, 
Department of Information and Computer Sciences, Nara Women's University, KitaUOYA Nishimachi, NARA 630-8506, JAPAN

E-mail address: yamasita@ics.nara-wu.ac.jp

Department of Mathematics, Kyushu University, 33, Fukuoka 812-8581 Japan

E-mail address: nishi@math.kyushu-u.ac.jp

Department of Mathematical and Computing Sciences, Tokyo Institute of Technology, Ohokayama, Meguro, TOKYo 152-8552 Japan

E-mail address: sadayosi@is.titech.ac.jp 